Cahiers d'études africaines

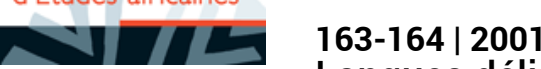

Langues déliées

\title{
À la frontière des langues
}

Figures de la démarcation

On the Borderline of Languages: Figures of Démarcation

\section{Cécile Canut}

\section{OpenEdition}

\section{Journals}

Édition électronique

URL : http://journals.openedition.org/etudesafricaines/104

DOI : 10.4000/etudesafricaines.104

ISSN : 1777-5353

Éditeur

Éditions de l'EHESS

Édition imprimée

Date de publication : 1 janvier 2001

Pagination : 443-464

ISBN : 978-2-7132-1394-6

ISSN : 0008-0055

Référence électronique

Cécile Canut, « À la frontière des langues », Cahiers d'études africaines [En ligne], 163-164 | 2001, mis en ligne le 21 novembre 2013, consulté le 05 février 2021. URL : http://journals.openedition.org/ etudesafricaines/104; DOI : https://doi.org/10.4000/etudesafricaines.104 


\section{Cécile Canut}

\section{À la frontière des langues}

Figures de la démarcation*

La réflexion proposée ici s'inscrit dans une perspective plurielle, à la fois linguistique, anthropologique et sociologique, ce que recouvre en partie la sociologie du langage. L'interdisciplinarité est nécessitée par l'objet même que je vais tenter de circonscrire, à savoir la nomination et plus particulièrement «l'enjeu de la nomination des langues ». Cette expression doit d'emblée être placée dans le sillage de l'ouvrage collectif «lancé » et dirigé par Andrée Tabouret-Keller, Le nom des langues, I. L'enjeu de la nomination des langues.

Plus que d'apporter des réponses, j'aimerais soulever un certain nombre de questions relatives au nom des langues en illustrant mon propos par différents exemples dont la majeure partie sera tirée du terrain africain et plus spécifiquement malien. Si j'ai déjà décrit brièvement les catégories linguistiques déterminant les différents termes utilisés pour nommer les langues au Mali, je voudrais maintenant engager une réflexion plus approfondie sur les motivations et les effets de la nomination, dans le prolongement de celle d'A. Tabouret-Keller : pourquoi donner des noms aux langues ? Qui nomme? Pour qui ? À qui ? Que fait-on quand on donne un nom (propre ou commun) à une langue ? Et surtout : faut-il que les langues aient un nom ?, question qui renvoie à l'excellente étude de P. Sériot (1997).

Autant d'interrogations qui impliquent la mise en cause, au niveau épistémologique, de la délimitation linguistique proposée par les linguistes euxmêmes. Se pose avant tout la question de la langue elle-même : qu'est-ce qu' une langue ? Y a-t-il de «vraies » et de « fausses » langues ? des «souslangues »? Une langue doit-elle être écrite, standardisée, pour avoir droit à un nom ou au statut de langue ?

Notre objectif, qui s'inscrit dans les perspectives de A. Tabouret-Keller et de J.-L. Amselle, sera de montrer que la nomination des langues, comme celle des communautés, résulte d'une construction sociale, d'une volonté d'homogénéisation notamment pour deux catégories de «donneurs de

* Une première version de ce texte, intitulée Le nom des langues ou les métaphores de la frontière, a été présentée à la Journée d'étude du CERCE sur les Représentations de l'autre, à l'Université de Montpellier III en mars 2000. 
noms »: les institutions (État, Justice, Église, etc.) et les linguistes, alors même qu'elle est pratiquée de manière beaucoup plus hétérogène et variante par les locuteurs, en fonction des situations.

S'il existe chez tout sujet le désir de tracer des frontières, de se représenter l'autre pour mieux s'en dissocier, ces dernières sont loin d'être équivalentes pour tous. Au contraire, on repère, pour un même locuteur, une grande fluctuation discursive et surtout interdiscursive (en fonction de l'autre, l'interlocuteur) qui se rattache à des fluctuations intersubjectives. Comme dans toute situation langagière, c'est le positionnement de soi par rapport à l'autre qui est en jeu et se manifeste par différentes distanciations dans les discours (Fénogio 1997 ; Gardy 1997) et une appropriation variante des discours de la doxa. Cette grande variation s'inscrit dans ce que Bakhtine appelle le dialogisme ou plus globalement l'interdiscursivité ${ }^{1}$, les discours antérieurs, le « déjà-dit » ou le « dit avant », « ailleurs », qu'ils soient politiques, idéologiques, sociaux, etc., parmi lesquels s'opèrent parfois des choix, et qui sont consciemment ou inconsciemment réactualisés dans nos propres discours. Ils sont bien souvent nourris d'habitus sociaux et culturels.

En fonction de l'ensemble de ces paramètres, on assiste à de multiples métaphores de la frontière, de la distance instaurée entre soi et l'autre, à des fins plus ou moins «identitaires ».

À travers diverses hypothèses quant à ce qui se joue dans la nomination, nous irons donc des discours les plus homogénéisants aux plus hétérogénéisants. Seuls les discours sont à prendre en compte, puisque la nomination s'inscrit dans un processus de construction socio-discursive.

\section{Nommer c'est faire exister, c'est construire}

La première question que je voudrais soumettre à l'analyse est : qu'est-ce que donner un nom à une langue ?

Avant de préciser les modalités de cette question (Qui nomme ? Qu'estce que l'on nomme ? Pour qui nomme-t-on ? à quelle fin ? etc.), il importe de savoir ce que l'on fait quand on donne un nom à une langue. Comme pour toute autre catégorie, J.-L. Amselle (1999) l'évoque pour les groupes et la notion d'ethnie : «[...] nommer c'est construire le groupe », donner un nom résulte d'un processus constructiviste : c'est faire exister une réalité qui ne l'était pas auparavant, c'est homogénéiser, clôturer un ensemble de réseaux ou d'éléments à l'origine en relation les uns aux autres de manière hétérogène. Ainsi, la délimitation entre le slovaque et le tchèque, autrefois considérés comme une même langue (tchécoslovaque en Tchécoslovaquie), ou celle entre le serbe et le croate (serbo-croate dans l'ancienne Yougoslavie) participe de cette volonté de tracer des frontières — d'ailleurs souvent

1. Construction du discours dans l'interaction langagière. 
similaires aux frontières politiques pour les noms de langues servant de causes nationales (nous y reviendrons).

C'est donc le rapport à l'autre — ou à soi en fonction de l'autre — qui est en jeu sous des formes imaginaires, fantasmées ou idéologisées : le nom n'existe que pour s'opposer à d'autres noms, c'est à l'autre qu'il s'adresse, c'est par rapport à l'autre qu'il se définit.

Nommer, c'est en quelque sorte construire et immédiatement figer ce construit tout en voulant le donner comme un réel, comme un objet du monde : « À partir du moment où une langue a un nom, elle devient un objet homogène, non plus un ensemble dans un diasystème, mais objet de politique linguistique, d'éducation, enjeu de la constitution d'un État-nation. Elle devient aussi, et surtout, objet de discours, qu'il est si facile de confondre avec un objet du monde » (Sériot 1997 : 167).

\section{Nommer c'est instituer socialement}

À la question : qui nomme ?, la réponse la plus fréquente dans les sociétés occidentales est : l'institution sociale (État, religion...), ou, par effet de retour, celui qui tente de contrer l'institution en s'y inscrivant fatalement (association de défense des langues minoritaires, groupes revendiquant la promotion des langues régionales en France, etc.).

Une des formes d'homogénéisation les plus fortes est en effet celle de l'institution (Tabouret-Keller 1997). Si Saussure donne une place institutionnelle majeure à la langue en tant que système linguistique arbitraire (institution pure), il ne la met pas au même niveau que les autres institutions sociales. Si cette singularisation est tout à fait discutable, d'après A. Tabouret-Keller, la nomination des langues est par contre un des déterminismes sociaux qui interviennent dans la construction et la manipulation des langues. Plus simplement, le nom, quel qu'il soit, attribue une place dans le réseau complexe des relations langagières. Lorsque les premiers colons ou administrateurs circonscrivent une langue ou un ensemble de langues et en inventent quasiment le nom - le maya pour les langues amérindiennes en Amérique centrale, le manding au Mali avec Delafosse —, ils procèdent à la fois à un découpage dans le champ langagier (le manding n'est pas le peul, le songhay, etc.) et à une homogénéisation (le malinké, le khassonké, le jula, etc., appartiennent à la même langue d'origine) : ils instituent socialement. Il n'y a donc jamais d'arbitraire du nom des langues. Même chez les linguistes (le bantou est par exemple une construction), les découpages sont au service d'un principe d'homogénéisation fondateur de la notion de langue, «en elle-même et pour elle-même », selon la célèbre phrase de Saussure.

Selon A. Tabouret-Keller (1997 : 9), il faut toutefois distinguer l'institution sociale des langues au sens des «conventions sociales » d'emplois de celle qui régit les structures politiques et sociales d'un État. Dans ce 
deuxième cas, le but des politiques est généralement de créer une langue unifiée, figée, standardisée, qui se donne comme "symbole et instrument de leur existence nationale » (Weinreich $1968:$ 648).

Le cas de la France est exemplaire: en inventant au $\mathrm{XVI}^{\mathrm{e}}$ puis au $\mathrm{XVII}^{\mathrm{e}}$ siècle une langue écrite, standard, prétendument destinée à contrer le latin, les institutions politiques françaises ont réussi, sous prétexte d'unification nationale, à anéantir la quasi-totalité des langues et parlers régionaux, faisant passer le français pour le langage maternel de tous les Français ${ }^{2}$. Mieux, en érigeant une seule norme d'usages (le bon usage, calqué sur l'écrit et utilisé uniquement par une élite) pour la langue parlée, elles sont parvenues à faire croire aux Français que ce français décrit en termes de pureté était la langue d'origine de la population alors qu'elle n'était qu'un des parlers d'Île-de-France, certes celui de la Cour et de la capitale. Ce double processus ${ }^{3}$ d'homogénéisation, anéantissement du plurilinguisme puis dévalorisation systématique de la variation au profit de la norme littéraire de l'élite, renforce le modèle de pureté attribué conjointement à la nation et à la langue.

Si le fantasme de pureté et d'unicité des langues est latent ou explicite dans tous les discours sur les langues, il apparaît ici concrétisé par l'idéologie nationale au point d'être institué socialement et politiquement: « Il existe des langues pures », toutes susceptibles d'être «contaminées » par d'autres idiomes. La légitimation politique de ce fantasme a donné lieu, au cours des siècles en France, à des mises en garde, voire des contre-attaques, multiples et répétées de la part de nombreux intellectuels (les puristes) à propos de la «dégradation» de la langue tour à tour «contaminée » par l'italien, l'anglais, l'arabe, etc. (Meschonnic 1997).

Pour faire face au centralisme français, des noms de langues sont alors créés selon le même schéma «constructiviste » comme celui d'occitan par exemple - qui n'a pas plus de réalité langagière que le françois et que les locuteurs des langues d'oc ont du mal à s'approprier ${ }^{4}$.

$\mathrm{Au}$ Maghreb, le même phénomène se produit avec la revendication berbère. L'ensemble des «parlers » berbères ne forment pas une langue berbère et les difficultés actuelles pour sa standardisation et son homogénéisation le montrent.

Les noms, d'où qu'ils viennent dans le champ social, sont créés pour instituer, légitimer soit une volonté politique (langue nationale à imposer) soit une réalité linguistique occultée (les langues exclues du domaine public et non reconnues par les politiques). L'impact sur les populations de ces manipulations, au niveau des représentations d'une part et des pratiques langagières d'autre part, est majeur. L'effet de feed-back des discours qui

2. Ordonnance de Villers-Cotterêts (1539).

3. Que P. ACHARD (1994) explicite dans ce qu'il appelle le double fonctionnement démique et ethnique.

4. Voir l'analyse de la biographie de Delteil (GARDY 1997). 
accompagnent ces décisions réside dans l'acceptation et parfois la soumission à de tels coups de force. Si des locuteurs (Occitans, Bretons, Basques, etc.) résistent encore dans les discours, et de moins en moins dans les faits, il est étonnant de voir comment en quelques siècles le nom même de français a pu générer un si fort «enthousiasme », puisque la plupart des locuteurs ont abandonné leur langue première au profit de cette langue d'État ${ }^{5}$.

De multiples explications comme l'urbanisation, la scolarisation, les guerres, l'extension de l'administration et de l'économie libérale, etc., permettent de comprendre en partie ce phénomène: au niveau symbolique comme au niveau pratique tout a été fait pour assurer l'homogénéisation linguistique totale du pays. Le français devient progressivement une langue emblématique qui, hors des frontières politiques, gage le bien-fondé des causes à défendre («langue de la liberté, de l'égalité, des droits de l'Homme »).

On assiste à une vision essentialiste des langues (une langue = une nation, une langue = une communauté voire une « ethnie ») alors même que nous savons combien les identités sont en perpétuelle négociation et que les pratiques ne cessent de varier et d'évoluer. Au figement du linguistique répond le figement des communautés, et vice versa.

\section{Nommer c'est dominer}

On vient de le voir avec le cas de la France, mais on pourrait en citer de multiples autres, les articles de ce volume le montrent à maintes reprises, la langue est un instrument de pouvoir et la nomination est un enjeu politique déterminant dans la construction des États-nations.

Si la nomination est l'affaire des législateurs et des savants puisque ce nom doit faire autorité (Tabouret-Keller 1997), l'imposition concerne non seulement un nom mais aussi l'appartenance à un groupe. Les manipulations idéologiques sont à ce niveau légion. Est-ce qu'une langue écrite a, elle seule, le droit à un nom, un nom propre ? Les dialectes et les patois ne seraient-ils pas de vraies langues pour ne posséder de la sorte qu'un nom commun globalisant?

La connotation implicite dans les choix des termes en France est révélatrice de cette hiérarchisation perpétuelle faite entre les langues et inscrite dans la langue elle-même. Ainsi, dans de nombreuses langues, notamment les langues d'Afrique de 1'Ouest, les termes correspondant à patois ou dialecte $^{6}$ n'existent pas. Il en existe par contre en portugais ou en arabe. Ce

5. Ainsi le breton n'est plus du tout transmis aux jeunes générations (LE BERRE \& LE DU 1997).

6. Les discours de certains intellectuels africains sur leurs langues, qui ne «sont que des dialectes bons à faire la cuisine et à chanter» (CANUT 1996), illustrent particulièrement l'effet de feedback étudié par J.-L. Amselle (1999): ces énoncés, ils ne peuvent les prononcer qu'en français car, dans leurs langues, le mot «dialecte» serait intraduisible... La hiérarchie ne s'opère jamais, dans les langues du Mali par exemple, en termes de valeurs intrinsèques aux langues et 
qu'il faut entendre à partir de l'appellation de « sous-langues » c'est qu'elles sont «sans grammaire », c'est-à-dire sans écriture. Toute langue possède une syntaxe, mais toutes ne sont pas instrumentalisées (normalisées et codifiées). Encore une fois, l'écriture devient, dans les discours, un phénomène constitutif de la langue alors qu'il ne résulte que d'une intervention humaine sur les langues, un constructivisme de plus. Certes dominante, notamment en Occident mais aussi en Afrique avec l'arabe (Amselle 2001), l'écriture ne doit rien à la langue : on peut écrire dans toutes les langues, mais on peut aussi parler, communiquer, sans passer par l'écriture.

La confusion entre syntaxe et grammaire écrite est vivement entretenue dans ce processus de dévalorisation de l'oral, de la variation et du mouvement. Les effets rétroactifs sur les discours sont d'ailleurs immédiats : en ce sens les nombreux parents maliens (et plus largement africains) qui refusent d'envoyer leurs enfants dans les écoles bilingues français/langue première (africaines) invoquent, entre autres, ces facteurs. Ils insistent sur le lien étroit entre école, français et écriture.

Dans le champ de la dévalorisation des langues et du plurilinguisme au nom du nationalisme, on peut aller jusqu'à nier l'existence et donc le nom d'une langue. C'est le cas du kurde en Turquie qui est systématiquement évincé dans les textes législatifs turcs (Akin 1997).

Si nommer c'est dominer, la situation de l'ancienne Yougoslavie est à cet égard exemplaire : le nationalisme est en prise directe avec la question des noms des langues. En dissociant une langue (serbo-croate) en trois langues (serbe, croate, bosniaque), les politiques illustrent en direct (il n'y a plus de métaphores ici) le processus d'homogénéisation. En tentant de trouver le maximum de différences entre les langues, ils renforcent leurs propres frontières politiques afin de créer des «États-nations ethniquement homogènes » (Djordjevic-Martinez 1999: 81). Ce qui fait dire à l'écrivain Vuk Draskovic que « le plus grand malheur pour les Serbes et les Croates est d'avoir une même langue » (cité in Djordjevic-Martinez 1999 : 87). Dans cette situation, les locuteurs, autrefois convaincus de parler la même langue avec des variations (comme partout ailleurs), se voient imposer des noms de langues qu'ils ne connaissent pas et qui ne servent ici que l'idéologie nationaliste. L'homogénéisation se renforce par une série d'interventions sur les langues : alors que les Serbes tentent de «serbiser» leur langue en évitant tout terme proche de la variété croate, en traduisant les œuvres littéraires croates, et en prônant l'alphabet cyrillique, les Bosniaques «arabisent » et «turkisent » leur langue... Les locuteurs, eux, se comprennent parfaitement, à moins qu'ils ne se soient déjà approprié ces nouveaux discours... Est-ce une situation?

Ces entreprises de «purification » des langues existent depuis longtemps et il serait erroné de les dissocier totalement de ce qui s'est passé en France

aucun nom explicite n'existe. Pour invoquer ce type de hiérarchie, les locuteurs passent nécessairement par une autre langue, et donc une autre vision du monde... 
au XVII ${ }^{\mathrm{e}}$ siècle (Meschonnic 1997); elles montrent en tout cas à quel point la langue et son nom sont un enjeu de pouvoir sans précédent dans l'espace public.

Sur la scène internationale, il serait aussi ambigu d'occulter l'enjeu de la nomination, l'exemple le plus frappant est celui de la francophonie. Il nous est impossible d'entrer dans les détails du rôle éminemment politique que joue «le français sans frontière », ce français soi-disant commun à l'espace dit «francophone », en Afrique notamment. En homogénéisant — quoi que puissent en dire les « francophonistes » se targuant depuis peu de prôner la diversité — un espace dit linguistique, alors que tout sépare les différents pays de la francophonie et la «langue» au premier chef, on impose un référent symbolique très fort qui fait du français une sorte d'emblème, de symbole international porteur de valeurs, etc. On fait porter à la langue des phénomènes historiques (Révolution française) et culturels (littérature, arts...). Au fond, ce que la francophonie permet de valoriser, au bout de la chaîne, ce n'est pas les pays en question mais le français-drapeau, les marchés économiques, l'implantation des entreprises françaises et les relations diplomatiques de la France ${ }^{7}$.

\section{Nommer c'est catégoriser}

J'en viens maintenant aux linguistes : sur quoi s'appuie leur choix de nomination ? Et avant tout, qu'est-ce qu'ils appellent une langue ? À partir de quand peut-on délimiter géographiquement une aire linguistique ?

Le travail des linguistes a longtemps été implicitement d'assigner une place aux langues, et de les hiérarchiser.

\section{La typologie des langues}

La question de la typologie des langues mériterait un long développement, nous renvoyons pour une étude approfondie au récent ouvrage de R. Nicolaï (2000) qui traite de cette problématique. Les linguistes procèdent depuis longtemps à des catégorisations, des hiérarchisations et des typologies. Les plus connues furent, en linguistique comparative et historique au XIX ${ }^{\mathrm{e}}$ siècle, les typologies élaborées en termes génétiques de filiation. Les nominations

7. L'implantation de la «francophonie » au Mozambique est de ce point de vue exemplaire. La construction d'un gigantesque centre culturel franco-mozambicain à Maputo, capitale lusophone où le français est totalement marginal, en dit long sur la volonté «francophone» de s'implanter dans la zone (entre l'Afrique de l'Est anglophone, l'Afrique des Grands Lacs convoitée et déstabilisée par les Américains, et l'Afrique du Sud en pleine expansion économique). Ces stratégies ne sont que les pâles répliques du libéralisme américain dont l'implantation est bien plus rapide et durable en Afrique. 
« langue-mère, langue-fille », etc., renvoyaient alors aux perspectives biologiques et évolutionnistes de l'époque (Schmitz à paraître). On a pu ensuite différencier les langues du monde en fonction de critères «linguistiques » différentiels et aboutir à des oppositions entre langues agglutinantes, langues flexionnelles, ou langues à tons (correspondance entre une famille de langue et un type linguistique). Une des dernières typologies fait état de cinq types de langues: type flexionnel externe, type flexionnel interne, type agglutinant, type polysynthétique et type isolant. Ces types sont distingués à partir de dominantes linguistiques et de rapports entre les formes (Pottier 1968 : 314). Si le travail typologique se poursuit, pour les langues indo-européennes mais aussi pour les langues africaines, et s'affine d'année en année, il reste que le phénomène lui-même et les représentations qui y sont engagées (la question de la « ressemblance » évoquée par Nicolaï par exemple) posent problème.

Un autre type de classification est celui qui fonctionne par aires géographiques. Quoi qu'il en soit, dans chaque cas il s'agit bien de tracer des frontières, de délimiter, de comparer et de hiérarchiser, et donc d'homogénéiser à partir de critères toujours très discutables.

\section{Les dialectologues}

Cette optique fut appliquée aux méthodes de la dialectologie qui ont révélé d'étonnants résultats. En voulant découper le territoire français par exemple selon les prononciations ou termes lexicaux (aires dialectales), les dialectologues se souciaient des dialectes, et donc apparemment de la variation. Si ce terme a été dissocié de son usage courant péjoratif («sous-langue »), ce type d'analyse ne fit pas l'économie d'une vision extrêmement ambiguë en termes d'opposition hiérarchique centre/périphérie (Léonard 1997). De plus, on peut remarquer dans l'objet même de ce travail la volonté d'homogénéisation toujours à l'œuvre, qui vient ici faire écho au centralisme français en matière de langue, et qui consiste à circonscrire des aires linguistiques homogènes. La variation serait-elle au fond suspecte pour qu'il faille ainsi la décrire dans le moindre de ses replis, pour qu'il faille la circonscrire et la réduire au maximum ?

Ainsi, J. Fourquet (1968: 574) explique : «[...] il y a des zones de transition où l'on ne peut tracer une limite que par une convention arbitraire, par exemple en convenant que la ligne qui sépare le type keval du type cheval sera réputée limite du picard et du champenois. [...] il fallut définir exactement le territoire de chacun pour éliminer les blancs. » Pourtant, soucieux de la frontière, les dialectologues continuent de chercher les critères scientifiques de délimitation des aires dialectales...

\section{La typologie des langues en sociolinguistique}

On pourrait croire que la sociolinguistique est un des domaines les moins marqués par les catégorisations linguistiques du fait même de son objet, les 
pratiques langagières et la variation. On se trompe, à quelques exceptions près ${ }^{8}$. Elle typologise autrement non plus les langues mais les variétés de langues.

Si la diversité est dans ce cadre acceptée, valorisée (au moins dans les discours, pas toujours dans les pratiques), elle reste sans cesse pensée, appréhendée à partir d'un socle originaire, d'un centre, d'un tout homogène. La variation, même chez Labov finalement, est dépendante d'un centre invariant, d'un système, alors qu'elle devrait être pensée comme constitutive des langues conçues non pas comme des systèmes clos mais comme des ensembles de diasystèmes en inter-relation.

J'ai fait à ce propos l'examen de la typologie des variétés et notamment l'usage des termes dialecte et créole par les sociolinguistes (Canut 2001). Il ressort que le terme dialecte, pour ne prendre que cet exemple, n'est d'aucune pertinence dans le champ de la sociolinguistique puisque du point de vue fonctionnel, il ne se différencie en rien de vernaculaire, terme largement utilisé par ailleurs. La permanence de dialecte est, au fond, le corollaire d'une autre permanence, souvent inconsciente et marquée par l'idéologie, celle d'une perception généalogique et monolithique des langues. Ainsi, le dialecte (lecte utilisé dans une zone géographique restreinte et uniquement en langue première, définition du vernaculaire) conserve le poids d'une hiérarchie constitutive, celle de sa provenance, de son origine. Le dialecte se différencie du vernaculaire du fait de son appartenance à une langue mère, c'est-à-dire de son inscription dans une "généalogie» linguistique. Cette appréhension verticale renvoie aux perspectives diachroniques et s'oppose donc radicalement à un positionnement fonctionnel revendiqué par les chercheurs. Ainsi, on distinguerait les langues qui ne sont pas issues d'autres langues, et les autres. Et, par conséquent, on pourrait en conclure que certaines langues ne s'inscrivent que dans une filiation, dénuées de tout contact avec d'autres langues. Il ne s'agit pas là d'occulter le rôle des études sur les mécanismes de transformation des systèmes en diachronie (propre au champ de la linguistique historique) mais de mettre le doigt sur les représentations des chercheurs qui occultent systématiquement la dimension du contact entre les langues dans le cadre du changement linguistique, dimension qui nous semble fondamentale pour la compréhension de ce phénomène ${ }^{9}$.

A l'inverse, le terme créole, dénué de toute filiation et renvoyant à la pluralité et à l'hétérogénéité maximale à l'origine, ne peut prétendre au rang de «langue». On continue, dans les typologies sociolinguistiques à le

8. Les sociologues du langage à partir des travaux de Gumperz: P. Wald, G. Manessy, C. Julliard, M. Dreyfus, M.-L. Moreau, ou R. Nicolaï, par exemple, sortent de ce cadre.

9. Nous suivons en ce point R. NiCOLAÏ (2000 : 191) qui préfère parler d' « héritage » plus que de «filiation » et indique que le modèle arborescent (ou l'arbre généalogique) traduit «non pas LA réalité mais UNE réalité construite», et qu'il y en a bien d'autres... 
considérer comme une variété à part (ni véhiculaire ? ni vernaculaire ?). Encore une fois, c'est sa constitution qui est en jeu et non son fonctionnement dans les pratiques langagières. Comme dans de nombreux cas - même si aujourd'hui beaucoup d'études sur les créoles ont dépassé cette vision —, le contact de langues dans la constitution des systèmes semble faire exception alors qu'il en est la règle. L'expression même de «langues en contact » (opposée à «langues sans contact» ?) est ambiguë. Elle suppose des systèmes homogènes coupés de la réalité des pratiques langagières mouvantes. Elle s'inscrit en fait dans une tradition historique construite autour du fantasme monolingue ${ }^{10}$ dont la portée est scientifiquement inefficace, d'autant plus dans les pays à fort plurilinguisme comme les pays africains où l'on ne peut en aucun cas faire abstraction du « contact».

\section{Sortir du structuralisme}

Si la sociolinguistique continue de penser uniquement en termes discontinus c'est, au fond, qu'elle n'est pas sortie du structuralisme. Que privilégier : un système de règles internes aux langues ou l'ensemble des relations et des variations langagières? (Amselle 1999: 54).

La notion de système implique la comparaison (ressemblances/différences entre les langues) alors que c'est l'ensemble des réseaux de relations entre les pratiques langagières qui sont à même de nous faire comprendre la complexité langagière.

La notion de langue en linguistique renvoie à un phénomène d'homogénéisation. Chez Saussure, la langue n'est plus conçue comme une substance (nouveauté essentielle au $\mathrm{XX}^{\mathrm{e}}$ siècle) mais comme une structure, « un tout interne de relations ». Si la linguistique s'est libérée du modèle «biologiste », elle demeure une science de l'homogène. La frontière posée par Saussure entre interne et externe, entre langue et parole, montre que la langue telle qu'elle est posée par les linguistes relève aussi d'une construction: «Elle révèle en particulier que le problème de la définition d'une langue, la plupart du temps prise comme un pur donné, reste le grand impensé de la linguistique » (Sériot 1997 : 168). En suivant d'autres auteurs (Berrendonner et al. 1983, Blanche-Benveniste 1997), il apparaît que la notion de système clos est inadéquate à la réalité des usages fluctuants repérables pour certains chercheurs en termes de «micro-systèmes » ou de « diasystèmes » (Léonard 1990).

Il nous faut montrer maintenant que la perspective épistémologique suivie ici provient et s'inscrit dans un long travail de terrain prenant en compte les dires, les paroles, des locuteurs. En effet, ceux-ci ne conçoivent pas les mêmes frontières que les linguistes ou les législateurs : leurs frontières sont

10. Sur la construction de ce fantasme judéo-chrétien, voir le chapitre «Babel et la confusion des cultures » (AMSELLE 2001). 
changeantes, floues, mouvantes... Ils font l'expérience permanente de la variation, à l'intérieur d'une même «langue », et entre les «langues ». Si la différence entre deux langues se fonde avant tout, pour eux, sur un critère d'intercompréhension, à cela s'ajoute ensuite bien entendu les effets de feedback des discours antérieurs, sociaux, politiques ou linguistiques réactualisés de manière fluctuante dans les interactions.

La polynomie des langues

« La polynomie est la règle plutôt qu'elle ne serait l'exception » (TabouretKeller 1997 : 9).

À la fluctuation de la perception de frontières est liée la fluctuation de la nomination. Les locuteurs utilisent une grande variété de noms pour les réalités langagières qui les entourent : le nom est fluctuant, la délimitation entre les langues est fluctuante, les identifications subjectives en matière de langues sont totalement fluctuantes.

TABLEAU RÉCAPITULATIF DES CRITÈRES DE DISTINCTION DES LOCUTEURS «MALINKÉ » (MALI, ZONE MANDINGUE-OUEST) DANS QUELQUES EXEMPLES DE NOMINATION DES LANGUES

\begin{tabular}{ll}
\hline \multicolumn{1}{c}{ Critères de distinction } & \multicolumn{1}{c}{ Nominations } \\
\hline 1. Lieux & $\begin{array}{l}\text { bamakokan* (langue de Bamako) } \\
\text { sagabarikan (langue de Sagabari) } \\
\text { kitakan (langue de Kita) } \\
\text { bakokan (langue de derrière le fleuve) }\end{array}$ \\
\hline 2. Sujets parlants (qualifiants) & $\begin{array}{l}\text { fakan (langue du père) } \\
\text { kanba (grosse langue) }\end{array}$ \\
\hline 3. Sociaux (groupes d'appartenance) & $\begin{array}{l}\text { wulamogokan (langue des paysans) } \\
\text { kokan (langue dans le dos = verlan) } \\
\text { konokan (langue des oiseaux) }\end{array}$ \\
\hline 4. Religieux & $\begin{array}{l}\text { banbaracinni (langue de païens pour } \\
\text { les Songhay) }\end{array}$ \\
\hline 5. Communautaires (ou « ethniques ») & $\begin{array}{l}\text { bamanankan (bambara), maningakan } \\
\text { (malinké) }\end{array}$ \\
\hline 6. Politiques & $\begin{array}{l}\text { langue officielle, langue nationale... } \\
\text { (en français) }\end{array}$ \\
\hline 7. Idéologiques \\
(feedback $>$ colonisation) & $\begin{array}{l}\text { dialectes (en français), manding } \\
\text { (groupe manding) }\end{array}$ \\
\hline 8. Linguistiques & $\begin{array}{l}\text { langue véhiculaire, vernaculaire, pidgin, } \\
\text { créoles (en français) }\end{array}$ \\
\hline
\end{tabular}

$*$ Kan $=$ «langue » ou «parler ».** Invention de langue type « javanais » chez les jeunes filles à Bamako. 
Ce que les locuteurs perçoivent ${ }^{11}$ comme «la langue de l'autre » n'est jamais définitivement établi. Au Mali, et plus particulièrement en zone dite « mandingue-est» (région de Kita), les délimitations entre les langues, nommées bambara et malinké par les linguistes, varient. D’une part ces termes ne recouvrent que rarement ceux des locuteurs qui proposent des différenciations plus complexes (bamakokan, kitakan, sagabarikan, etc.) souvent fondées sur les distinctions géographiques, d'autre part, la généralisation « mandingue »s'exprime uniquement en fonction de la position symbolique occupée par le locuteur à un moment donné de son discours et réactualisant d'autres discours. Le tableau ci-dessous schématise quelques positionnements discursifs déterminants des noms de langues (de soi ou des autres). Nous les présentons graduellement en fonction de l'impact des discours antérieurs sur les langues.

Outre ces premiers critères grossièrement présentés, une multitude de fluctuations intersubjectives vient affiner le phénomène de construction de la nomination. Ainsi à Kita, un grand nombre de jeunes qualifient leur parler de «bamanankan » non pas en référence au groupe des Bambara ${ }^{12}$ mais en lien avec leur désir d'appartenance (symbolique) à un autre lieu, la capitale, considéré comme «moderne ». Par conséquent, la langue est pour eux porteuse de cette « modernité » et l'appropriation du nom, même face à l'enquêteur, leur permet de s'identifier à cet autre lieu, à cet autre qu'ils tendent à devenir. Par ailleurs, devant leurs parents ou grands-parents, leurs discours peuvent totalement changer (emploi de «kitakan, maningakan ») car leur positionnement intersubjectif n'est plus le même. La frontière qu'ils tracent est à la fois géographique et symbolique. En même temps, ils déclarent que «le bamanankan et le maninkakan c'est pareil mais c'est pas la même chose ». Ce discours récurrent dans les enquêtes montre, pour chaque lieu d'enquête, que les frontières sont mouvantes, que les délimitations ne sont pas seulement posées de l'extérieur mais soumises à l'investissement subjectif de chacun. Le « raidissement» des frontières et de la différence apparaît plus nettement à partir de l'actualisation des discours sociaux, ethniques et politiques, encore que dans le cas des langues au Mali, aucune véritable politique officielle de différenciation ne soit en place $^{13}$ (le malinké comme

11. Ces remarques proviennent d'enquêtes de terrain composées d'observations et d'entretiens à propos des pratiques langagières et des langues (en 1996 puis 1998).

12. La référence ethnique est loin d'être prépondérante. Les mêmes distorsions entre langue et identité dite «ethnique » sont repérées par F. DEJou (1999) en zone minyanka.

13. Toutefois, dans les faits, tant du point de vue social que politique, la fonction du bambara se distingue de celle des autres langues nationales (langue véhiculaire, langue associée à la modernité, à la capitale, etc.). La diffusion du bambara dans les zones non bambarophones à travers la politique de mutation des fonctionnaires (enseignants, policiers, etc.) n'est pas sans effet sur la répartition actuelle du paysage sociolinguistique malien. 
le bambara sont des langues «nationales » face au français, langue officielle). Ceci explique l'extrême labilité des positionnements. Le discours généralisant sur le manding en tant qu'unité linguistique homogène est assez peu représenté à travers le terme «manding » (quelques intellectuels). Par contre, la référence historique et culturelle à une origine commune entre les Malinkés est davantage présente.

\section{Les négociations intersubjectives dans le rapport aux langues}

L'identification à une langue n'est pas une donnée définitive, close, immuable. Comme l'évoquait une locutrice peule : «Quand je suis à Paris, je suis Malienne, quand je suis à Bamako je suis Peule et quand je suis dans mon village, je suis Peule du Guimbala. » De fait, la langue, et même la langue «du père », de la lignée, est loin d'être assignée à une identité unique et homogène. F. Dejou (1999) identifie les mêmes fluctuations en zone minyanka lorsque à la question «Quelle langue parlez-vous ? , un locuteur peul répond: "Le bambara bien sûr, nous sommes Fulas, pas Minyankas!»

Ce qui détermine l'énonciation de ces différents positionnements, c'est à la fois le lieu et l'interlocuteur. Les catégorisations s'opèrent en fonction de la relation à l'autre : vis-à-vis d'un Français, on se définit «nationalement» alors que dans son village, les catégories implicites communes à tous (malienne, peule) sont inopérantes. Au-delà de cet aspect pragmatique, s'ajoutent les désirs d'appartenance (géographique, sociale, ethnique, politique, etc.) qui entraînent des négociations permanentes dans la relation aux langues. Les frontières, dans cette complexité relationnelle, ne se donnent pas de l'intérieur mais résultent de fluctuations intersubjectives: elles se construisent et se déconstruisent en permanence.

Pour revenir au rôle des chercheurs, lorsque Calvet (1996) se demande si, à propos du serbo-croate, le linguiste doit considérer qu'il s'agit d'une langue ou de deux langues, il est évident que cette question est inopérante et encore très marquée par le structuralisme. Il pose la langue comme une donnée du réel en écartant la subjectivité comme élément constitutif du rapport au langage. Puisque les noms et donc les délimitations ne sont que le produit de discours et de constructions, rien dans le réel ne peut constituer les limites d'une langue (Achard 1994 : 68). Le linguiste ne peut rien en dire, il ne peut que mettre au jour les mécanismes complexes de ces constructions, à la fois dans les discours et dans la diversité des pratiques.

D'une part, il faut tenir compte des discours épilinguistiques des institutions (puristes, politiques, nationalistes ainsi que ceux des locuteurs qui inscrivent leurs propres discours dans ces optiques) afin d'expliciter le phénomène $\mathrm{d}^{\text {' }}$ "homogénéisation ${ }^{14}$ et de découpage légitimé : les méta-

14. De l'interpénétration de ces différents discours résulte parfois (et de plus en plus aujourd'hui) ce que l'on peut observer aussi dans le champ anthropologique avec la notion d'ethnie: renvendications identitaires, associations immédiates entre 
phores de la frontière élaborées à différents degrés et renvoyant au mythe de l'unité et de l'origine. Dans ce cas, un nom renvoie toujours à une langue en discours (la partie pour le tout ou le tout pour la partie).

D'autre part, il est nécessaire de prendre en compte l'autre partie des discours des locuteurs, discours les moins imprégnés de la doxa, les plus subjectifs et les plus hétérogénéisants ${ }^{15}$, afin de concevoir, comme pour tout autre espace culturel, que les pratiques langagières relèvent d'un large continuum, un réseau de pratiques langagières imbriquées les unes dans les autres et qu'il ne convient pas de distinguer des «langues» différentes. Ces discours appartiennent au pôle hétérogénéisation et adviennent, plus ou moins rapidement selon les cas, lors des entretiens (Canut 2000). Ainsi, alors qu'au Mali les locuteurs, notamment en zone rurale, sont moins soumis à un appareil discursif métalinguistique fort, les discours de type hétérogénéisant sont plus fréquents, plus spontanés. À l'opposé, en France, où les discours sur la langue se multiplient depuis des siècles, un travail de subjectivation est nécessaire pour les locuteurs monolingues soumis à la pression centralisatrice et normative extérieure.

\section{Nommer c'est s'inscrire entre les groupes}

En fonction des interactions, les locuteurs choisissent un nom, en créent, se positionnent socialement, discursivement. Mais ce positionnement n'est jamais stable ni définitif. Il varie en fonction de deux paramètres : la relation entretenue par le locuteur avec son interlocuteur (groupe, âge, sexe, position politique, profession, imaginaire, etc.) et avec les discours antérieurs (discours puriste, «ethnique », discours national, régional, pour la défense des langues minoritaires, etc.)

S'il y a bien toujours une volonté de s'inscrire dans un groupe à un moment donné, le positionnement est toujours fluctuant et varie car la constitution des groupes elle-même varie. Il est évident qu'en fonction du

langue et identité, ou toutes formes de différenciations et de radicalisation des frontières. On observe ces renforcements identitaires en ville (association de défense de la langue et la culture peule à Bamako par exemple), des lieux où les «particularismes locaux s'inscrivent toujours dans le cadre d'un système plus vaste qui leur donne sens » (AMSELLE 2001: 50). À l'inverse, dans les villages peuls du Guimbala, les jeunes refusent d'être alphabétisés en peul mais préfèrent le bambara. On le voit, la «substancialisation» des langues ou leur « ethnisisation » ne s'opère que dans un resserrement identitaire et donc dans l'opposition à l'autre : "L'identité implique d'emblée une traduction et une conversion parce qu'elle est un être pour les autres » (ibid. : 59).

15. L'étude de S. BenNIs (dans ce numéro), montre que plus les sujets sont engagés dans des processus de socialisation (réseaux de type professionel, institutionnel, religieux, politique, associatif, scolaire, etc.) plus leurs discours sont marqués par les idéologies propres à ces différents réseaux. À l'inverse, dans les villages les plus isolés, les discours sont davantage marqués par l'hétérogénéité et l'ambivalence. 
durcissement des discours sociaux (homogénéisation forte des communautés, discours «ethniques », imposition de frontières politiques, ou sociales, renforcement des identités, etc.) les positionnements sont de plus en plus dépendants de la multiplication des frontières et de leur légitimation sociopolitique. Face au nationalisme serbe, les croates optent irrémédiablement pour un discours nationaliste, le seul possible pour répondre à un autre nationalisme, en radicalisant de fait le processus d'unification et de différenciation.

Au-delà de cette pression discursive, un certain nombre de qualités imaginaires sont attribuées aux langues en fonction de l'autre, celui qui la parle. C'est dans les pratiques langagières que se construit l'image de l'autre mais cette image n'est jamais fixe, immuable. La preuve en est apportée par les mélanges, des mixtes linguistiques, qui loin d'être des exceptions ou des cas particuliers, sont la réalité première des pratiques langagières (Canut 2001).

\section{Quand les frontières tombent: les mixtes langagiers}

Une réalité permanente en Afrique et ailleurs et qui vient contredire la dimension essentiellement homogène dans la caractérisation des langues ou la volonté de tracer des frontières, est bien le mélange des langues. Phénomène très développé dans les sociétés d'Afrique de l'Ouest, mais pas seulement $^{16}$, il vient démontrer que la notion de délimitation en matière de nom de langue n'est pas opérante. En effet, les mélanges, dans les discours, ne sont pas systématiquement dévalorisés et prennent dans les usages une dimension essentielle. Ils n'ont pas, pour les locuteurs, de réels noms (sauf ceux que les sociolinguistes leur donnent et qu'ils réactualisent par effet de feed back comme le pidgin english au Cameroun ou au Nigeria) mais une multitude d'appellations en fonction des situations.

Le cas du parler d'Abidjan, étudié par K. Ploog (2000) et nommé par elle «abidjanais » est symptomatique de notre problématique. Si les locuteurs, à la question de la nomination de leur parler, déclarent qu'il s'agit de français ${ }^{17}$, c'est qu'ils s'inscrivent non pas dans le cadre de l'homogène (ou de la rupture, ou encore de la différence ou différenciation des parlers) mais dans l'indistinction des frontières, le continu, signifiant par là même l'hétérogénéité constitutive des langues. S'ils différencient bien leur français du

16. Tout locuteur bilingue connaît cette pratique, mais tout locuteur monolingue aussi.

17. Les catégorisations à ce propos ne manquent pas (bon/mauvais français, par exemple) mais correspondent à des processus connus (CANUT 1996, 2000) de hiérarchisations des variétés et entraînent inévitablement les divergences de positionnement épilinguistiques chez les locuteurs. Ce qui est intéressant dans le travail de K. Ploog concerne la nomination par les jeunes («français») alors même qu'ils constatent les difficultés de comprénhension entre eux et leur interlocutrice française... On est ici à la limite du seuil de la délimitation généralement opérée par les locuteurs entre deux langues. 
français de l'école par exemple, ils le font à travers des critères évaluatifs (bon/mauvais), indiquant alors qu'à l'intérieur d'une langue, il y a tout cela : les mélanges, les variations, les transformations, les appropriations. Que doit alors faire le linguiste face à une telle situation ? Faut-il nécessairement qu'il tranche en faveur de critères dits «linguistiques » pour faire de ce parler une langue à part entière et lui donner un nom ? Dans ce cas, il s'inscrit lui-même, inconsciemment parfois, dans un positionnement idéologique (la reconnaissance d'un parler, d'une «langue à part entière » face au français). Ce procédé de légitimation d'une variété dévalorisée devenue «langue » provient souvent des chercheurs occidentaux qui tentent peutêtre aussi de se départir du système de catégorisation et de hiérarchisation des parlers et des langues, inscrit dans les langues occidentales mêmes (poids des «valeurs» attribuées aux langues: bonne/mauvaise, vraie/ fausse...). En voulant ainsi revaloriser les langues, ils instituent des frontières avant la lettre, ou plus, imposent des différenciations que les locuteurs eux-mêmes n'auraient peut-être jamais produites, mais qu'ils revendiqueront par contre à la suite des chercheurs en retour. Ils se placent irrémédiablement dans le discontinu.

Le choix le moins marqué sera peut-être de respecter les termes utilisés par les locuteurs à chaque phase de l'évolution des parlers, tout en décrivant les modalités de la variation tant dans les usages que dans les discours épilinguistiques.

Les mixtes linguistiques montrent en tout cas à quel point le rapport des locuteurs aux langues est constitutivement hétérogène et variant. La variabilité au niveau des usages, des nominations et de la perception des métissages, le prouve.

Quelle conclusion tirer de tout cela ? Faut-il pour autant éviter le terme « langue »? S'il fallait éviter, dans le cadre des discours scientifiques, tous les termes à connotation idéologique, ils n'en resteraient plus beaucoup ! Il convient tout d'abord d'écouter les locuteurs: suivre leurs propres frontières, leurs propres nominations. Cela éviterait aux linguistes de poser des catégories ad hoc dont la visée n'est autre que la recherche d'une clôture, d'une origine (Marcel d'Ans 1997) et la création de communautés sociolinguistiques qui n'existent pas en elles-mêmes. S'appuyer sur les dires des locuteurs ne signifie pas que la réflexion se fige à leurs propos, l'intrusion dans les champs de l'anthropologie, de la sociologie, etc., nous aide précisément à construire l'interprétation la plus complète possible afin de démêler l'ensemble des paramètres assurant la construction des discours. En retraçant systématiquement les processus sociaux, symboliques, politiques et idéologiques en œuvre dans l'élaboration des « frontières » linguistiques, on libère alors l'étude des usages et des pratiques en ne les enfermant plus dans une langue définie a priori $^{18}$. L'approche du langage, ou des pratiques

18. «En d'autres termes, il s'agirait d'inverser le raisonnement habituel qui consiste à opposer radicalement universalisme et relativisme afin de montrer que l'univer- 
langagières, ne s'opèrent plus en termes de structure ou même de variation ${ }^{19}$, elle réside dans le décryptage de fluctuations liées les unes aux autres au sein de réseaux perméables et entremêlés. À partir du «continu » des usages, peuvent se dégager ensuite toutes les constructions homogénéisantes, toutes les formes figées par des frontières, résultant systématiquement des discours posés sur la diversité et la pluralité langagières.

Le travail que je mène sur les discours dits «épilinguistiques » (discours des locuteurs sur le langage, les langues, les pratiques langagières) m'a amenée à repérer que la grande fluctuation interdiscursive qui régit les paroles s'inscrit en fait dans une fluctuation intersubjective fondatrice du rapport du sujet au langage. Cette seconde fluctuation se caractérise par une tension perpétuelle entre homogénéisation et hétérogénéisation, entre des figures de l'homogène, de l'unité, de l'UN, donc de la frontière, et des figures de l'hétérogène, de la diversité, de la pluralité. Ces ambivalences incluent bien évidemment la nomination des langues qui sont autant de métaphores de ce jeu entre les deux pôles. La reconnaissance de l'autre en soi, de l'hétérogénéité comme constitutive du sujet amène les locuteurs à se défaire, dans les discours les plus subjectifs, de ce qui se donne dans la clôture, la fermeture, l'homogénéité, l'exhibition des frontières, la mesure de la différence, etc., et qui métaphorise au plus haut point la figure mythique de l'origine, d'une langue originaire, fixe, immuable, pure, et dotée d'un nom!

La langue est introuvable, inappropriable parce que justement, à travers les usages, elle se donne à la fois dans la pluralité et le mouvement et dans le fantasme de sa clôture.

Pour nous, l'essentiel serait donc de rejeter toutes catégorisations extérieures à celles, fluctuantes, des locuteurs eux-mêmes pris dans le flot des autres discours. Si « le discontinu est bien le produit de la défaite du continu » (Amselle 2001: 183), il revient au linguiste de ne pas tomber luimême dans le piège du discontinu en reproduisant le schéma homogénéisant propre aux discours de la frontière quels qu'ils soient. Tout en repérant l'interaction de ces différents positionnements, il s'agit de se départir, dans l'analyse, de tout constructivisme a priori (langue, communauté, système, ethnie...) et donc de rejeter toute structuration interne de la langue comme objet clos puisque la langue n'existe que dans la configuration optimale

salisme, loin de contrarier la manifestation des différences, est le moyen privilégié de leur expression» (AMSELle 2001: 49).

19. La notion de «variation » est elle-même extrêmement ambiguë puisqu'elle réfère inévitablement à un centre, un système homogène donné comme objectivement invariant à partir duquel les pratiques varient. 
d'un ensemble de réseaux en contact. Le «syncrétisme originaire » (Amselle 1999) reste pour nous la seule hypothèse à défendre, en anthropologie comme en sciences du langage.

LACIS, Université Paul Valéry-Montpellier III.

\section{BIBLIOGR APHIE}

ACHARD, P.

1993 Sociologie du langage, Paris, PUF («Que sais-je ?»).

1994 «Constitution des États-nations et des langues», Langues et identités, Cahiers de la francophonie, 2, Haut Conseil de la francophonie : 63-69.

AKIN, S.

1997 «Désignation d'une langue innommable dans un texte de loi : le cas du kurde dans les textes législatifs turcs », in A. TABOURET-KeLLER, ed., Le nom des langues, I. Les enjeux de la nomination des langues, Louvain-La-Neuve, Peeters-Bibliothèque des Cahiers de l'Institut linguistique de Louvain-95: 69-80.

Amselle, J.-L.

1999 Logiques métisses, Paris, Payot.

2001 Branchements. Anthropologie de l'universalité des cultures, Paris, Flammarion.

Amselle, J.-L. \& Sibeud, E. (dir.).

1998 Maurice Delafosse. Entre orientalisme et ethnographie : l'itinéraire d'un africaniste, Paris, Maisonneuve \& Larose.

AuthieR-Revuz, J.

1995 Ces mots qui ne vont pas de soi. Boucles réflexives et non-coüncidence du dire, 2 vols, Paris, Larousse.

BAKHTINE, M.

1977 [1929] Le marxisme et la philosophie du langage, Paris, Éditions de Minuit.

BERRENDONNER, A., et al.

1983 Principe de grammaire polylectale, Lyon, Presses universitaires.

Blanche-Benveniste, C.

1990 «Grammaire première et grammaire seconde : l'emploi de EN », Recherche sur le français parlé, $10: 51-73$.

1997 «La notion de variation syntaxique dans la langue parlée», in F. GADET, Langue française. La variation en syntaxe, Paris, Larousse : 9-29. 
BRANCA-RoSOFF, S.

1996 «Les imaginaires de la langue », in H. BOYER, ed., Sociolinguistique. Territoire et objets, Paris, Delachaux et Niestlé : 79-114.

Bronckart, J.-P., Perrenoud, P. \& Schoeni, G.

1988 La langue française est-elle gouvernable? Normes et activités langagières, Neuchâtel-Paris, Delachaux \& Niestlé.

Calvet, L.-J.

1996 «Une ou deux langues? Ou le rôle des représentations dans l'évaluation des situations linguistiques », Études créoles, XIX (2) : 69-82.

CANUT, C.

1996 Dynamiques linguistiques au Mali, Paris, Didier Érudition.

2000 «Subjectivité, imaginaires et fantasmes des langues : la mise en discours "épilinguistique" ", Langage et Société, 93: 71-100.

2001 «Créoles et dialectes, la typologie des variétés face aux dires des locuteurs », communication au colloque du LACIS, juin 2000, Université Montpellier III, Traverses, 2 : 387-410.

À paraître «Perceptions of Languages in the Mandingo Region of Mali », in D. R. Preston, ed., Handbook of Perceptual Dialectology, II, Amsterdam, John Benjamins.

Dejou, F.

1998-1999 Variation sur un même terme: les usages de la désignation bamanafla au Minyankala (sud-est du Mali), Mémoire de DEA, Paris, EHESS.

DJORDJEVIC-MARTINEZ, K.

1999 Politiques linguistiques dans "l'espace yougoslave», la linguistique dans l'ombre de la politique, Mémoire de DEA, Montpellier, Université Paul Valéry-Montpellier III.

FÉNOGIO, I.

1997 «Parler une langue, dire son nom », in A. TABouret-Keller, ed., op. cit. : 241-250.

FourQueT, J.

1968 «Langue-dialecte-patois », in A. Martinet, Le langage, Paris, Encyclopédie de la Pléiade : 571-596.

GARDY, P.

1997 «Nommer l'occitan? À propos d'un récit mythique de nomination », in A. TABouret-Keller, ed., op. cit. : 251-270.

LE BERRE, Y. \& LE Dû, J.

1997 «Ce que nomme "breton"», in A. TABOuRET-KelLER, ed., op. cit. : 99-116.

LÉONARD, J.-L.

1990 Variation dialectale et microcosme anthropologique : l'île de Noirmoutier (Vendée, France), Thèse de doctorat, Aix-en-Provence, Université de Provence. 
1991 «Dialecte, distance et représentations ethnolinguistiques », in J.-C. BouviER, ed., Les Français et leurs langues, Aix-en-Provence, Publications de 1'Université de Provence : 59-83.

1997 «Diasystème et diglossie périphérique : le cas gallo-roman », Colloque $Y$ a-t-il une exception sociolinguistique française?, Brest, Université de Brest.

Le Page, R. \& Tabouret-Keller, A.

1985 Acts of Identity, Creole-based Approaches to Language and Ethnicity, Cambridge, Cambridge University Press.

Manessy, G. \& WALD, P.

1979 Plurilinguisme: normes, situations, stratégies, Paris, L'Harmattan.

1984 Le français en Afrique noire, tel qu'on le parle, tel qu'on le dit, Paris, L'Harmattan-Ideric.

MARCEl D'Ans, A.

1997 «Les anciens Maya ne parlaient pas le maya ! Considérations sur la nomination des langues indiennes en Hispano-Amérique », in A. TABOURET-Keller, ed., op. cit. : 191-224.

MeschonNic, H.

1997 De la langue française, Paris, Hachette.

NicolaÏ, R.

2000 La traversée de l'empirique. Essai d'épistémologie sur la construction des représentations de l'évolution des langues, Paris, Ophrys.

PloOG, K.

2000 La syntaxe du premier actant : entre contraintes morphosyntaxiques et élaboration discursive. Étude d'un corpus parlé abidjanais, Carnet de grammaire, Toulouse-Le Mirail, Rapport interne de l'Équipe de recherche en syntaxe et sémantique.

PotTier, B.

1968 «La typologie», in A. Martinet, Le langage, Paris, Encyclopédie de la Pléiade : 300-324.

PRIEUR, J.-M.

1996 Le vent traversier. Langage et subjectivité, Montpellier, Presses de l'Université de Montpellier («Langages et cultures»).

Schmitz, J.

À paraître Races de langues. Les voyages de la culture (Antiquité, Afrique, Inde).

SÉRIOT, P.

1997 «Faut-il que les langues aient un nom ? », in A. TABOURET-KelLER, ed., op. cit. : $167-190$.

TABouret-Keller, A.

1988/1997 «Contacts de langues : deux modèles du XIX ${ }^{\mathrm{e}}$ siècle et leurs rejetons aujourd'hui », in A. TABOURET-Keller, La maison du langage, Montpellier, Presses de l’Université de Montpellier («Langages et cultures») : 97-114. 
1989 «À l'inverse de la clarté, l'obscurité des langages hybrides », Revue de l'Institut de sociologie, numéro spécial, Le concept de clarté dans les langues et particulièrement en français, 1-2 : 19-29.

TABOURET-Keller, A., ed.

1997 Le nom des langues, I. Les enjeux de la nomination des langues, LouvainLa-Neuve, Peeters-Bibliothèque des Cahiers de l'Institut de linguistique de Louvain-95.

WALD, P.

1990 «Catégories de locuteur et catégories de langue », Langage et Société, 52 : $5-22$.

1994 «L'appropriation du français en Afrique noire : une dynamique discursive », Langue française, 104 : 115-124.

WEINREICH, U.

1968 Language in Contact, Findings and Problems, La Haye-Paris, Mouton.

\section{RÉSUMÉ}

À partir du phénomène de nomination des langues et en fonction des différents types de discours épilinguistiques (locuteurs, institutions, idéologie, etc.), cet article tente de montrer combien la notion de "langue», en linguistique, est une construction calquée, de par l'histoire occidentale de cette discipline, sur un modèle homogénéisant dominant. À la lumière de la polynomie constante présente chez les locuteurs maliens, on observe combien le besoin de nommer s'inscrit dans un processus sans cesse en tension dans les discours entre hétérogénéisation et homogénéisation, entre ouverture à la pluralité et repli sur l'origine perçue comme unité. Le nom vient circonscrire des frontières, imaginaires ou construites à partir d'autres champs : frontières sociales, politiques, idéologiques, religieuses, etc.

\section{ABSTRACT}

On the Borderline of Languages: Figures of Demarcation. - Given the phenomenon of naming languages and different types of epilinguistic speech (speakers, institutions, ideology, etc.), the effort is made to show that the notion of "language" in linguistics has been contructed owing to this discipline's history in the West on the model of a homogenizing dominant power. The constant "polynomy" among Malians sheds light on the need to name as part of a permanently tense speech process between heterogenization and homogenization, between openness to plurality and withdrawal toward an origin perceived as a unity. Names mark borders, whether imaginary or constructed on other social, political, ideological, religious, etc. grounds.

Mots-clés/Keywords : Mali, mandingue, continu/discontinu, discours épilinguistique, frontière, identités, langue, nomination/Mali, Manding, epilinguistic speech, borders, identity, language, naming. 